\title{
Emprendimiento social para el desarrollo comunitario en México: el caso del centro de desarrollo comunitario sustentable (CEDECOSU) del CREFAL en Pátzcuaro, Michoacán ${ }^{1}$
}

\author{
Miguel Rodrigo González Ibarra²
}

\begin{abstract}
Resumen
Este artículo tiene como objetivos analizar cuál es la importancia del emprendimiento social para el desarrollo comunitario y destacar cuál fue la experiencia del Centro de Desarrollo Comunitario Sustentable (CEDECOSU) del CREFAL, ubicado en Pátzcuaro estado de Michoacán, México, para la construcción de proyectos de emprendimiento y participación educativa. A través de un enfoque de investigación cualitativa por medio del análisis teórico y la sistematización empírica de los proyectos Haz crecer tú comunidad, Gestión del Desarrollo Comunitario y Producción Orgánica Sustentable entre 2012 y 2014, se deduce que el CEDECOSU se instituyó como motor para impulsar iniciativas desde la comunidad para enfrentar los problemas de exclusión social y educativa con base a los principios de economía social y solidaria.
\end{abstract}

Palabras clave: Emprendimiento Social, desarrollo comunitario, Centro de Desarrollo Comunitario Sustentable, CREFAL-Michoacán.

\section{Empreendedorismo social para o desenvolvimento comunitário no México: o caso do Centro de Desenvolvimento Comunitário Sustentável (CEDECOSU) do CREFAL em Pátzcuaro, Michoacán.}

\section{Resumo}

O objetivo deste artigo é analisar a importância do empreendedorismo social para - desenvolvimento da comunidade e destacar a experiência do Centro de Desenvolvimento Comunitário Sustentável do CREFAL (CEDECOSU), localizado em Pátzcuaro, no estado de Michoacán, México, para a construção de projetos comunitários, empreendedorismo e participação educacional. Por meio de uma abordagem qualitativa de pesquisa, por meio de análise teórica e sistematização empírica dos projetos Crescer sua comunidade, Gestão do desenvolvimento comunitário e Produção orgânica sustentável entre 2012 e 2014, segue-se que o

\footnotetext{
${ }^{1}$ Agradezco a la Dra. Ilse Brunner Schoenemann y al Mtro. Víctor Manuel Beltrán Morales del CREFAL por la oportunidad para conocer el programa, pero, sobre todo, por compartir su convicción y perspectiva de vida al servicio de la comunidad.

${ }^{2}$ Doctor en Ciencias Políticas y Sociales por la Universidad Nacional Autónoma de México (UNAM). Profesor-Investigador en el Departamento de Sociología de la Universidad Autónoma Metropolitana-Iztapalapa (UAMI), Ciudad de México. Orcid Id: https://orcid.org/0000-0003-3999-3532. E-mail: ibarra000@yahoo.com.
} 
CEDECOSU foi instituído como motor para promover iniciativas da comunidade para enfrentar os problemas de exclusão social e educacional baseados nos princípios da economia social e solidária.

Palavras-chave: Empreendedorismo Social, Desenvolvimento comunitário, Centro de Desenvolvimento Comunitário Sustentável, CREFAL-Michoacán.

\title{
Social entrepreneurship for community development in Mexico: the case of The Center for Sustainable Community Development (CEDECOSU) of CREFAL in Pátzcuaro, Michoacán.
}

\begin{abstract}
The purpose of this article is to analyze the importance of social entrepreneurship for community development and highlight the experience of CREFAL's Center for Sustainable Community Development (CEDECOSU), located in Pátzcuaro state of Michoacán, Mexico, for the construction of community projects. entrepreneurship and educational participation. Through a qualitative research approach through theoretical analysis and empirical systematization of the projects Grow your community, Community Development Management and Sustainable Organic Production between 2012 and 2014, it follows that CEDECOSU was instituted as a motor to promote initiatives from the community to face the problems of social and educational exclusion based on the principles of social and solidarity economy.
\end{abstract}

Key words: Social Entrepreneurship, community development, Sustainable Community Development Center, CREFAL-Michoacán.

\section{Introducción}

Como producto de la crisis económica registrada en la primera mitad de la década de los años noventa del siglo pasado en la mayor parte de los países latinoamericanos, especialmente en México, se incrementó la posibilidad de articular formas de emprendimiento y organización alternativas para impulsar el desarrollo comunitario y enfrentar los problemas asociados con la pobreza, el desempleo y la exclusión en el ámbito rural (Kliksberg, 2015:2).

El emprendimiento tiene muchas interpretaciones y una de ellas es la de emprendedurismo que refiere a la construcción de proyectos para la creación de empresas bajo un enfoque solidario, sostenible y productivo para impulsar el desarrollo comunitario (CASTELAR, 2016:349). En este proceso 
se enlaza la noción de desarrollo comunitario para comprender aquellas iniciativas de las comunidades en diferentes áreas del desarrollo social, económico, humano y cultural que tienen como finalidad realizar cambios en sus condiciones de vida, fomentar el bienestar y lograr la inclusión social en el espacio público y político.

En el Estado de Michoacán, específicamente en la región lacustre de Pátzcuaro, existen iniciativas generadas por grupos, organizaciones e instituciones que han trabajado en la construcción de políticas y proyectos productivos para resarcir los efectos de la crisis económica y los problemas sociales vinculados a la falta de empleo, pobreza y exclusión de la población en las actividades económicas y productivas locales (BELTRÁN, BRUNNER, 2014:45-48).

Hacia el final de 2011, el CENTRO DE COOPERACIÓN REGIONAL PARA LA EDUCACIÓN DE ADULTOS (CREFAL en adelante), constituyó el Programa Michoacán Letrado (PML) para contribuir a la educación popular y coadyuvar en la creación de empresas sociales para contribuir a la calidad de vida de las comunidades más vulnerables del Estado. El programa inició con un proyecto piloto para la formación de empresas solidarias y la formación educativa de jóvenes y adultos en esta región lacustre a través de la formación del CENTRO DE DESARROLLO COMUNITARIO SUSTENTABLE (CEDECOSU).

Este artículo tiene como objetivo analizar cuál es la importancia del emprendimiento social para el desarrollo comunitario y destacar cuál fue la experiencia del CEDECOSU para la construcción de proyectos de emprendimiento y fomentar la participación social y educativa. Como hipótesis consideramos que esta institución se planteó como reto la coordinación para el desarrollo de proyectos para emprender la creación de empresas sociales y motivar el cambio social a través de la participación comunitaria. Los proyectos Haz crecer tú comunidad, Gestión del Desarrollo Comunitario y Producción Orgánica Sustentable desarrollados entre 2012 y 2014, revelaron la necesidad de establecer mecanismos de coordinación interinstitucional para fomentar la formación educativa y establecer una 
política integral para la creación de empresas sociales con base a los principios de economía social y solidaria.

Para el desarrollo de este trabajo se utilizó un enfoque de investigación cualitativa a través de la revisión de documentos institucionales, análisis de los proyectos antes referidos y sistematización de información para contextualizar y realizar una explicación sobre los resultados obtenidos hacia el desarrollo comunitario en la región. Asimismo, se utilizaron informes de gobierno sobre las actividades socioeconómicas en que se localiza el caso de estudio.

Así, este artículo se organiza en tres secciones. En la primera parte, se realiza una discusión crítica sobre la relación entre emprendimiento y el desarrollo comunitario, y se plantea una perspectiva sobre la importancia de la coordinación entre actores sociales e institucionales con respecto al desarrollo local. En la segunda, se contextualiza la gestación del Programa Michoacán Letrado y se analizan algunas de las iniciativas generadas desde el CREFAL para impulsar una agenda política más amplia hacia el tema en esta región. En la tercera parte, se exponen los objetivos, estrategias y desarrollo de los proyectos, así como se presentan algunos resultados alcanzados hacia la participación social.

Finalmente, se realizan una serie de reflexiones finales sobre los desafíos y oportunidades que se observaron en la construcción de proyectos sociales para el desarrollo comunitário, así como las implicaciones sociopolíticas y educativas que limitan los mecanismos para generar el emprendedurismo desde una perspectiva más amplia e integral en esta región lacustre afectada por la crisis económica en el estado de Michoacán.

\section{Emprendimiento para el desarrollo comunitário}

De inicio, las nociones de emprendimiento y comunidad son categorías conceptuales interdependientes en la medida de que se construyen en y para la comunidad. Asimismo, el emprendimiento tiene una conexión directa con el crecimiento económico y con la definición de 
políticas públicas hacia la innovación social y la formación de empresas solidarias (CASTELAR, 2016:351).

En este marco, y en el contexto de los impactos que se presentaron por las políticas de ajuste económico de tipo neoliberal durante la década de los años noventa en la mayor parte de los países de la región latinoamericana, especialmente hacia la reducción del gasto público, la focalización de programas sociales y las limitaciones de la inversión pública en comunidades rurales (LEÓN, 2014:19-37), el emprendimiento se puede considerar, por un lado, como una estrategia alternativa para motivar el desarrollo comunitario y mitigar los problemas sociales y, por el otro, como un modelo para construir procesos de aprendizaje colectivo y descentralizado en las comunidades.

Una persona emprendedora es aquella que facilita no sólo procesos de innovación social y empresarial, sino promueve cambios para la generación de proyectos y métodos alternativos de producción de bienes y/o servicios (CHONG-GONZÁLEZ, 2017:4). Una variante del emprendimiento es el emprendedurismo que refiere a la definición de espacios e identificación de oportunidades de trabajo en la comunidad y que tiene como misión coadyuvar al nivel de vida de las personas excluidas o desatendido por el mercado de bienes y servicios dirigidas por instituciones del sector público y privado. Si bien existe una reflexión acerca de la importancia de la presencia de un agente innovador que crea soluciones hacia diferentes problemas que enfrenta la sociedad y que puede producir cambio social, no existe un consenso único para establecer criterios para la formación de empresas sociales (CHALCATL, 2015:5).

En esta discusión, el emprendimiento en lo social se desarrolla como parte de un proceso más amplio y su misión es la búsqueda de oportunidades para determinar nuevos bienes y servicios, materias primas y métodos de organización. El emprendimiento advierte la necesidad de construir y evaluar oportunidades para un cambio social. Ciertamente, el rol del emprendedor (agente) es central en el proceso de construcción de políticas y programas sociales. De igual modo, el emprendimiento propone la 
definición de capacidades para generar una solución a un problema social y desde un enfoque eficiente, sustentable y con valor público (PALACIOS, 2010:581).

Otra discusión importante es aquella que establece que el emprendimiento social forma parte de una nueva ruralidad que se expresa en los sistemas económicos y que, como producto de la globalización, el uso de las tecnologías y los cambios en las formas de vida, entre otros factores, supone otra forma de vivir y generar mecanismos integración comunitaria (CHONG-GONZÁLEZ, 2017:31). A este respecto la economía social y solidaria supone la necesidad de identificar las bases materiales para el desarrollo personal social y ambiental del ser humano, así como analizar el impulso a los valores universales como la justicia, equidad, fraternidad, solidaridad y democracia directa que se tornan fundamentales para analizar los procesos de interacción social.

Desde otra perspectiva se considera que la economía social hace referencia a la resignificación de valores humanos y principios de solidaridad, que propugnan el reconocimiento de la otra persona como fundamento de la acción humana y eje de la renovación de la política, la economía y la sociedad (CORAGGIO, 2008:2). Así, se infiere la importancia que tiene la creación de actividades y organizaciones de carácter comunitario, asociativo, cooperativo, mutualista y otras formas de acción colectiva que se dirigen hacia la formación de empleo y la incidencia para incentivar el aprendizaje colectivo.

A este respecto, podemos expresar que las acciones de emprendedurismo requieren no sólo la participación de esfuerzos individuales, sino advierten la construcción de procesos de coordinación social e institucional basadas en el intercambio de conocimiento, recursos e información. Coincidimos con MONZÓN y DEFOURNY (1992:287), cuando analizan que estas acciones crean incentivos para avanzar hacia el establecimiento de una estrategia de desarrollo más amplia por medio de empresas o instituciones regidas por un funcionamiento democrático y solidario y avanzar en la satisfacción de las necesidades de la comunidad. 
Sea como fuere, la creación de un modelo de organización o empresa solidaria contribuye a la formación de capital social adaptándose al mismo tiempo al mercado y motivando la creación de formas alternativas de trabajo y aprendizaje colectivo frente a la crisis que experimenta el manejo de la política estatal hacia el desarrollo local. Lo local, con su escala próxima y su complejidad social, tiende a reconfigurarse como una nueva referencia ante el contexto de una economía debilitada y el rediseño de las políticas de globalización en la economía mundial (BOUZADA,1995:2-3).

En este orden de ideas, consideramos que la aparición de nuevos agentes -en tanto sujetos sociales transformadores- en el seno de las comunidades propicia la oportunidad para generar, impulsar y redescubrir las prácticas de la economía solidaria e impulsar la participación colectiva, la cooperación, autogestión, democracia y conservación del equilibrio de los ecosistemas. Además, las prácticas de la economía social detonan la colaboración entre grupos, organizaciones e instituciones para la formación de centros de aprendizaje y capacitación en el tema.

Ahora bien, y como se planteó anteriormente, el desarrollo de la economía solidaria se vincula inexorablemente con el desarrollo comunitario o local. Aunque existe un cierto debate sobre las contradicciones que tiene el desarrollo hacia lo social, cultural y económico, es importante discutir que la construcción de una política integral sugiere la identificación de cambios cuantitativos y transformaciones cualitativas bajo un equilibrio en todos los sectores y aspectos de la vida social manteniéndose a lo largo del tiempo (BAÑOS, 2012:25).

El desarrollo advierte lograr la satisfacción de necesidades básicas, así como articular a los individuos no sólo con la naturaleza, la tecnología y los procesos globales, sino propiciar una relación con lo colectivo, la planificación con la autonomía y la articulación de la sociedad civil con el Estado (Inchaustegui, 2000:172). Por su parte, el desarrollo humano se concibe como un proceso orientado hacia la creación de las condiciones para que los individuos, grupos y comunidades propicien cambios en la actitud y hábitos, así como se generen las escenarios para impulsar sus 
capacidades y habilidades analíticas y creativas que favorezcan sus objetivos y metas en sus procesos de crecimiento integral.

La asociación de los individuos, como sujetos, se torna fundamental para generar ideas y compartir experiencias que les permitan arribar hacia la construcción de proyectos educativos en el ámbito de sus condiciones históricas y sociales en que se desarrollan. Desde esta perspectiva, considero que la participación social no es posible sin la comprensión y la voluntad de actuar en comunidad. Una comunidad puede definirse como:

\footnotetext{
"una agrupación de personas que habitan un espacio geográfico delimitado; en el cual sus miembros tienen conciencia de pertenencia, existe interacción entre sí a través de diversos medios con el objetivo de alcanzar la satisfacción de sus necesidades, resolver problemas o desempeñar funciones relevantes a nivel local (ANDER-EGG, 2003:27)".
}

En síntesis, y por un lado, la discusión acerca del emprendimiento social y solidario conlleva a examinar las condiciones locales, regionales y globales en que se sitúa una determinada comunidad y, por otro lado, explica la importancia que tiene la construcción de proyectos de desarrollo comunitario bajo un método de intervención específico para propiciar en los sujetos un sentido de responsabilidad y solidaridad para la identificación y reflexión de sus problemas particulares y con la finalidad de promover el conocimiento y la participación en los procesos de intervención. Se puede aseverar que la comunidad es el ambiente micro-social donde la cooperación, el intercambio y la ayuda mutua, entre otros factores, aportan a la creación de los procedimientos de gestión, programas y proyectos hasta alcanzar el nivel macrosocial.

También es importante subrayar que la relación entre el emprendimiento y el desarrollo comunitario se legitima desde la comunidad como ámbito de convivencia, aprendizaje y coordinación para el desarrollo y es la base para la construcción de alternativas para el desarrollo comunitario (BAÑOS, 2012:12). La importancia de lo local radica en la actividad de los actores, de sus interrelaciones, conflictos, acuerdos y medios 
de cooperación encaminados hacia la transformación de la realidad para mejorar sus condiciones de vida. La comunidad es provista de una dinámica cultural e identidad colectiva en donde las decisiones de los actores son la base para la generación de alternativas y soluciones en los ámbitos micro y macro; de lo local a lo global.

Asimismo, cuando aludimos al desarrollo se observa un proceso complejo que tiene como objetivos crear las condiciones de progreso económico y social para la comunidad. Cabe recordar que la ORGANIZACIÓN DE LAS NACIONES UNIDAS (ONU) en 1963 definió al desarrollo comunitario como un espacio fundamental para el crecimiento económico y social en el ámbito local, especialmente en aquellas zonas rurales aisladas y que requieren de una mejor comunicación entre el gobierno y sociedad y para la formación de capital social ante las nuevas circunstancias (ANDER-EGG, 2000).

\section{Contexto y constitución del cedecosu en Pátzcuaro, Michoacán}

En las últimas dos décadas en México los procesos de reforma económica y política generados desde el Estado han discutido una agenda política para vigorizar el desarrollo local, a través de la descentralización de los procesos productivos y el apoyo a la creación de empresas sociales en aquellos lugares donde se registran altos niveles de pobreza, marginación y exclusión social (MORENO; ROS, 2010:274-292). En este sentido, el trabajo de organismos de cooperación regional y educativo (como el CREFAL), así como el trabajo de organizaciones de la sociedad civil se vislumbra valioso para la implementación de programas y proyectos hacia el desarrollo comunitario.

Aunque la construcción de esta agenda demanda una mayor investigación sobre los procesos de movilización social hacia el gobierno, el análisis acerca del desarrollo comunitario plantea un diagnóstico acerca de las formas de organización comunitaria para la ejercer la intervención y desarrollar mecanismos para el trabajo voluntario que permitan impulsar, 
poco a poco, la participación social y generar aprendizaje que permitan la incubación de nuevos proyectos y evaluar los impactos obtenidos.

De acuerdo con el Plan de Gobierno 2015-2021, en el estado de Michoacán se requiere impulsar el desarrollo productivo y fortalecer las políticas públicas para la generación de empleo y la organización social (Gobierno del Estado de Michoacán, 2015). Esta entidad se encuentra ubicada en el oeste de la República Mexicana y cuenta con poco más de 580 mil habitantes integrados en 113 municipios y organizados en 11 regiones. La entidad registra un $69 \%$ de población urbana y $31 \%$ rural; el $59.62 \%$ de la población se encuentra en situación de pobreza y $11.35 \%$ padece pobreza extrema y existe una población indígena importante conocida como la región purépecha (INEGI, 2020; véase FIGURA 1).

FIGURA 1. Michoacán y la región Pátzcuaro-Zirahuén

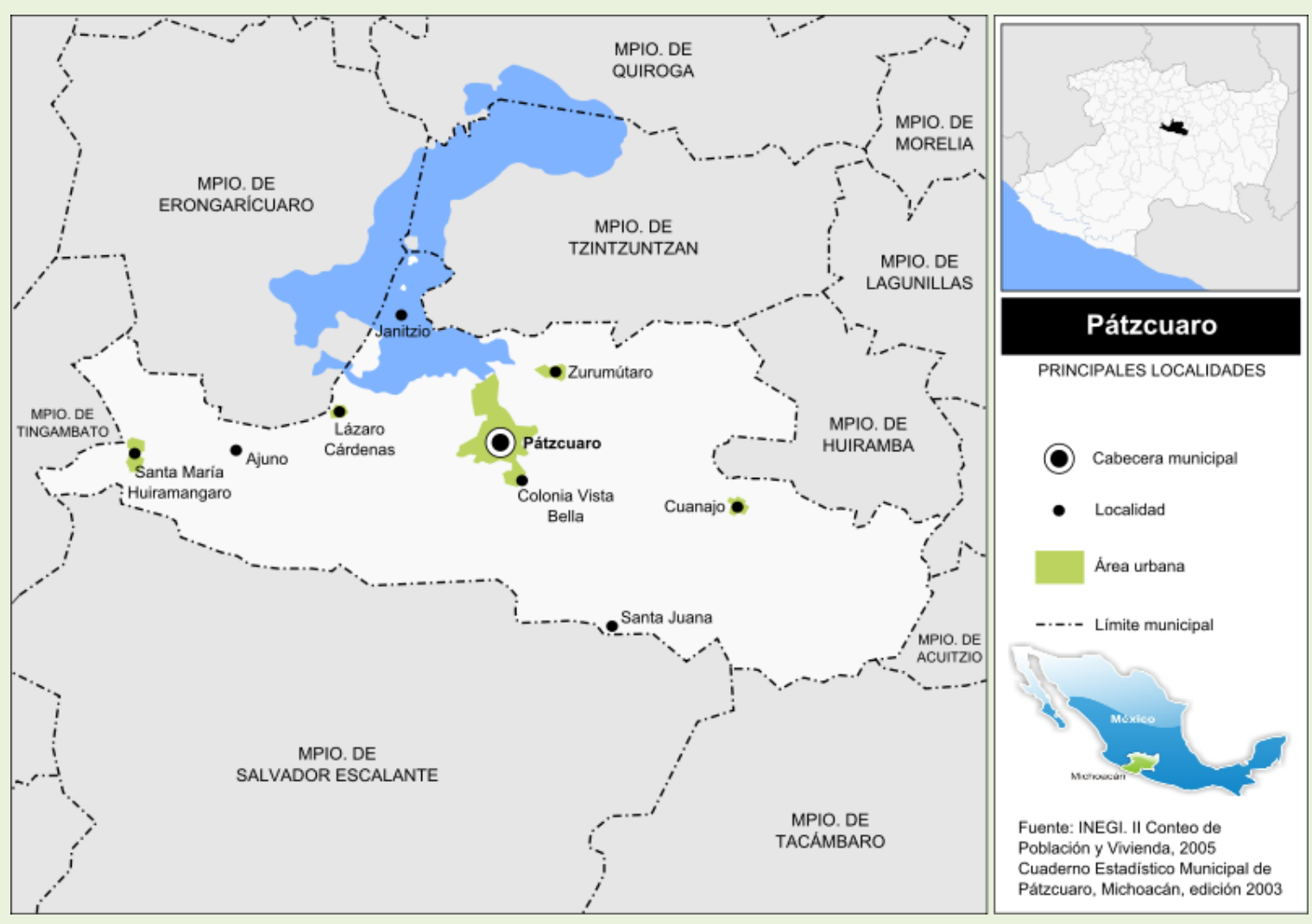

Fuente: INSTITUTO NACIONAL DE GEOGRAFÍA Y ESTADÍSTICA (INEGI), 2020. 
En el mismo PLAN DE DESARROLLO ESTATAL (2015:12) se propone una visión incluyente y sustentable para el crecimiento y que busque atender los problemas como la inseguridad, la falta de empleo, la escasa cobertura educativa y de deficiencia de los servicios de salud pública. Entre las prioridades de la agenda de gobierno se encuentran impulsar el desarrollo familiar y la inversión productiva para generar trabajo, así como preservar y acrecentar los valores de las familias. De la misma forma, se plantea que es necesario cubrir las necesidades básicas y generar inclusión, lograr la prevención del delito, fomentar la innovación y productividad; así como el desarrollo humano, el acceso a la educación y la salud.

De estas prioridades, la pobreza en todas sus formas se puede considerar como el principal desafío ya que más del 50\% de la población registra carencias y 14 de cada 100 habitantes se caracterizan por registrar pobreza extrema e insuficiencia alimentaria. En el ámbito educativo, los indicadores advierten una situación deficiente y que afectan desde el 2012 , a poco más del $26.1 \%$ de la población total antes mencionada. Los municipios con mayor número de personas con la carencia educativa son Morelia (capital del estado), Uruapan, Zitácuaro, Zamora y Apatzingán (GOBIERNO DEL ESTADO DE MICHOACÁN, 2015).

Por ejemplo, en la región de la Meseta Purépecha integrada por los municipios de Charapan, Cherán, Chilchota, Nahuatzen, Nuevo Parangaricutiro, Paracho, Tancítaro, Taretan, Tingambato, Uruapan y Ziracuaretiro, se registra que el $69.23 \%$ de la población se encuentra en situación de pobreza, donde el $24.37 \%$ tiene pobreza extrema muy por arriba de la media estatal. Más de la mitad de esta región se ubica en la ciudad de Uruapan. El $10 \%$ de la población habita en comunidades indígenas y más del $50 \%$ de la producción se obtiene del cultivo del aguacate. En la región existe la necesidad de crear proyectos productivos, tecnificación de agua de riego, creación de granjas lombrícolas y fomento al cultivo de maíz criollo, impulsar las actividades de traspatio, granjas piscícolas y repoblamiento bovino y porcino, así como la capacitación artesanal para auto emplear a 
jóvenes y adultos sin profesión o desempleados (GOBIERNO DEL ESTADO DE MICHOACÁN, 2015, Región 6).

Por otra parte, en la región nombrada como tierra caliente, integrada por los municipios de Carácuaro, Huetamo, Madero, Nocupétaro, San Lucas, Tacámbaro y Turicato, los datos indican que el $32.74 \%$ de la población se registra pobreza extrema y un alto índice de marginación social pese a que esta zona se caracteriza por una alta producción de aguacate, caña de azúcar, mango y cultivo de autoconsumo. El municipio de Huetamo es el que registra el mayor problema de desempleo y falta de inversión en las comunidades, así como la escases de proyectos productivos, infraestructura de servicios de salud, deterioro de la calidad de vida y mejora de planteles educativos, entre otros problemas de narcotráfico y violaciones a los derechos humanos (GOBIERNO DEL ESTADO DE MICHOACÁN, 2015, Región 8).

Ahora bien, en la región conocida como Pátzcuaro-Zirahuén el panorama no es menor, ya que esta zona registra un alto número de demandas y conflictos relacionados con la falta de desarrollo económico, inversión y empleo digno. En especial en los municipios de Pátzcuaro, Tzintzuntzan, Salvador Escalante, Quiroga, Pátzcuaro, Lagunillas, Huiramba y Erongarícuaro se concentra el mayor número de demandas estatales, así como se detecta poco más del $65 \%$ de la población en situación de pobreza. Si bien la artesanía, el cultivo de aguacate, la pesca y el turismo son las mayor fuente de ingresos, los retos esenciales que se detectan son la creación de empresas sociales y proyectos productivos integrales, así como el desarrollo de alternativas para el manejo de los recursos naturales y programas que atiendan las necesidades de las comunidades. Destaca el fortalecimiento de procesos de organización social para generar nuevas formas de interlocución con los programas sociales para evitar el control social desmedido, así como valorar su sistema de gobierno basado en usos y costumbres (GOBIERNO DEL ESTADO DE MICHOACÁN, 2015, Región 8).

En síntesis, las principales propuestas de carácter socioeconómico en la agenda política estatal tienen que ver con acciones relacionadas con el 
emprendimiento social y solidario. Asimismo, se plantea la consideración de espacios para la comercialización y promoción a nivel nacional e internacional de las artesanías de la región; acceso a créditos blandos, dirigido a los artesanos; programas de capacitación y asistencia técnica para la elaboración de artesanías administradas por las propias comunidades; apoyo para maquinaria especializada para sus cultivos y programas que otorguen semillas mejoradas; creación de programas sustentables para la región y el cuidado de los recursos naturales, entre otros acerca de la capacitación y concientización en temas ambientales y mayor colaboración de los tres órdenes de gobierno y de la ciudadanía para vigilar la aplicación de las normas y reglamentos en la materia.

En esta explicación, cabe recordar que la Ley de la Economía Social y Solidaria en México (DIARIO OFICIAL DE LA FEDERACIÓN, 2015:2) tiene por objeto establecer los mecanismos para fomentar el desarrollo y dar visibilidad de la actividad económica del sector social de la economía. Asimismo, esta ésta se prevé definir las reglas para la promoción y fomento del desarrollo social y económico del país, la generación de fuentes de trabajo digno, el fortalecimiento de la democracia y lograr la equitativa distribución del ingreso y contribuir al patrimonio social.

Consideramos que el análisis sobre la actividad socioeconómica en Michoacán subraya la urgencia para impulsar procesos de emprendimiento social y solidario ante el vacío gubernamental hacia esta región. Asimismo, se deriva que es necesario la creación de capital social, y visualizar una estructura para evitar la falta de integración social en las regiones, municipios y localidades con el propósito de generar oportunidades para la introducción de bienes y servicios, materias primas y métodos de organización a través de la creación de alianzas y programas sociales para la creación de oportunidades para el cambio social.

Precisamente, en el contexto de la región Pátzcuaro-Zirahuén se coloca el surgimiento del Centro de Desarrollo Comunitario Sustentable (CEDECOSU) a través de la creación del Programa Michoacán Letrado impulsado por el CENTRO DE COOPERACIÓN REGIONAL PARA LA 
EDUCACIÓN DE ADULTOS EN AMÉRICA LATINA Y EL CARIBE (CREFAL) entre 2012 y 2015. En efecto, desde la década de los años cincuenta del siglo anterior, el CREFAL fue impulsando la formación de promotores para el desarrollo comunitario y estableció programas de enseñanza-aprendizaje relacionados con la higiene, salud, economía familiar, cursos de alfabetización y educación básica para jóvenes y adultos.

Si bien el contexto sociodemográfico y económico ha cambiado, el CREFAL conservó entre sus prioridades impulsar el desarrollo comunitario y asignó al CEDECOSU la misión de ofrecer servicios de formación y apoyo para la creación y consolidación de conocimientos, habilidades y actitudes en jóvenes y adultos de las comunidades rurales y periurbanas en la búsqueda de una vida digna, mediante la generación de una cultura emprendedora, a partir de la creación de negocios de bienes y servicios que beneficien a las comunidades más vulnerables y que promuevan un desarrollo sustentable en toda la región latinoamericana. Entre los principios de trabajo se consideró impulsar el enfoque de la sustentabilidad con la finalidad de establecer una perspectiva multidimensional entre los procesos sociales y el medio ambiente bajo un modelo sustentable para mejorar la calidad de vida y la productividad de las personas, de las comunidades y sus zonas de influencia (BELTRÁN, BRUNNER, 2014:47).

A nivel operativo, con el establecimiento del CEDECOSU, el programa propuso la generación de proyectos a través de una articulación de esfuerzos, sociales e institucionales, para incidir en áreas dañadas por el analfabetismo y los déficits educativos, desde el bajo rendimiento escolar y la deserción, hasta las afecciones a la salud, la degradación ambiental, el desempleo y la carencia de prospectivas para una vida digna y de participación ciudadana (CREFAL, 2012:3).

El funcionamiento del CEDECOSU inició con varias iniciativas generadas para obtener financiamiento de la Embajada Alemana, la CONFEDERACIÓN ALEMANA DE COOPERATIVAS (DGRV) y la colaboración del Programa de Educación Inicial No-Escolarizada del CONSEJO NACIONAL DE FOMENTO EDUCATIVO (CONAFE) del gobierno federal, así como la 
colaboración de los municipios de la cuenca del Lago de Pátzcuaro. Asimismo, se pensó lograr la incorporación de nuevas tecnologías para actualizar y mejorar la infraestructura de servicios en la región Pátzcuaro y establecer mecanismos de coordinación con programas sociales gubernamentales, organizaciones de la sociedad civil y comunitarias.

La metodología de trabajo (véase FIGURA 2) esencialmente se fundamentó en un trabajo de reflexión y colaboración utilizando teorías, aportes en grupo y realimentación de las actividades y prácticas desde la comunidad. La tesis central de programa fue impulsar prácticas y procedimientos sistematizados para mantener, modificar y transformar aspectos centrales de la realidad social. Así, se promovió un modelo procesual basado en la premisa "De la ida a la difusión masiva" en los procesos de enseñanza aprendizaje y con una finalidad pedagógica y de construcción del desarrollo comunitario. Asimismo, este modelo planteó una enfoque exploratorio desde una fase del escalamiento gradual por medio de la difusión controlada en diferentes ámbitos comunitarios y para lograr la promoción de la difusión masiva asistida por el CREFAL no sólo en México sino para la región de América Latina y el Caribe (BELTRÁN, 2015:3)

FIGURA 2. Modelo de aprendizaje para el emprendimiento social
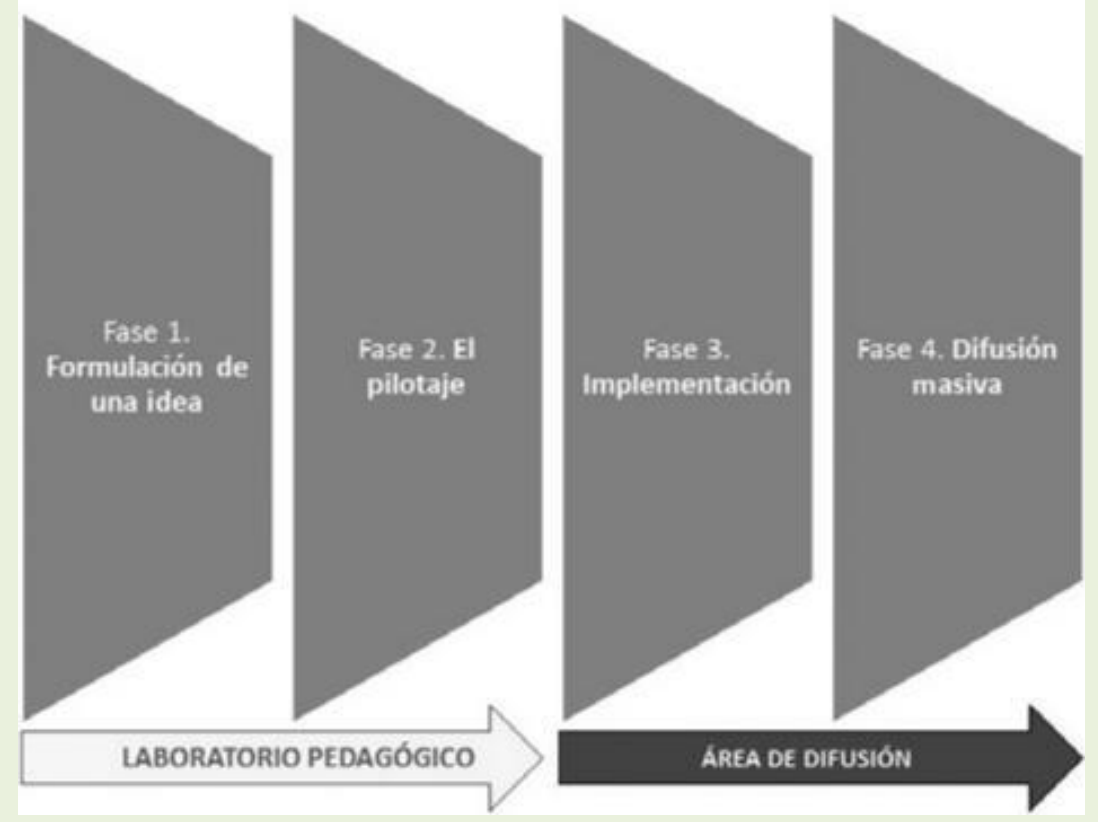

Fuente: BELTRÁN; BRUNNER, 2014. 
En síntesis, el programa propuso realizar un diagnóstico específico para cada situación (proyecto comunitario) en el que se reconocieran los problemas, las necesidades para fomentar el desarrollo de la comunidad. De esta manera, se estableció un laboratorio pedagógico para impulsar los intervenciones e iniciar con la implementación de tres proyectos socioeducativos: Haz crecer tú comunidad, Gestión del Desarrollo Comunitario y Producción Orgánica Sustentable.

\section{Proyectos hacia el emprendimiento social y el desarrollo comunitário}

\section{Haz crecer a tu comunidade}

El proyecto conocido como Haz Crecer a tu Comunidad, se concibió en 2012 como diplomado, y fue dirigido a la formación de grupos de jóvenes mujeres con el deseo de crear proyectos productivos y convertirse en microempresas solidarias que les proporciona empleo, alimentación y salud. Como objetivos del diplomado se planteó desarrollar trabajo en equipo y apoyo mutuo; impulsar el compartir saberes entre las personas y grupos; llevar a cabo aprendizajes prácticos y con referencias teóricas; utilizar los aprendizajes en la realidad cotidiana; y realizar proyectos con base en una metodología de intervención-acción y trabajo participativo (CREFAL, 2011).

La primera fase de sensibilización, inició con una campaña de información sobre el diplomado en 50 de las comunidades más apartadas vulnerables de los Municipios de Pátzcuaro, Salvador Escalante, Tzintzuntzan, Erongarícuaro y Quiroga y dio inicio a un taller de motivación para la formación de Cooperativas Productivas impartido por instructores de la Sociedad Cooperativa Agropecuaria Regional Tosepan Titataniske del Estado de Puebla, la Unión Regional de Apoyo Campesino URAC del Estado de Tlaxcala y personal de la Confederación Alemana de Cooperativas (DGRV).

En este taller se registró una afluencia importante con 220 personas de 30 comunidades a los cuales se hizo la invitación a participar en el 
diplomado Haz Crecer tu Comunidad. Los requisitos para formar parte del programa incluyeron ser un grupo constituido por jóvenes de 14 a 29 años y/o mujeres de cualquier edad, tener la idea de un proyecto con un alto valor para su comunidad y con probabilidad de éxito y tener como grupo un alto nivel de aceptación por parte de las autoridades y las familias en la comunidad. Asimismo, se impartió un taller sobre la estructura organizacional de una cooperativa y se dio a conocer el programa del diplomado organizado en seis módulos de clases presenciales para por lo menos cuatro integrantes de cada grupo

En una segunda fase, se llevó a cabo la capacitación para el desarrollo del proyecto para 14 grupos de trabajo (véase CUADRO 1). Si bien en las primeras sesiones existió una asistencia importante, se detectó que muchos de los participantes no lograron continuar debido a la falta de recursos para transporte al CREFAL y otros desertaron por la falta de comprensión al tema, así como por la dificultad en el lenguaje con los instructores emprendedores. El ejercicio principal se basó en compartir entre los asistentes su experiencia y pensar tareas colectivamente. Cada módulo se organizó en nueve horas, divididas en dos fines de semana por mes, un viernes en la tarde y el siguiente sábado en la mañana. Los temas que se abordaron en el diplomado fueron acerca de la viabilidad del proyecto, diseño operativo, mercadeo, plan estratégico, finanzas y contabilidad y figuras asociativas. Al concluir, la capacitación se realizó una feria comunitaria para dar a conocer los proyectos y buscar atraer el interés de aliados e inversionistas solidarios.

La tercera etapa fue de evaluación de resultados. Una vez que concluyó la feria, se brindó la oportunidad a los grupos de presentar sus planes de negocio al Centro de Innovación y Apoyo de Negocios (CIAN) del Instituto Tecnológico Superior de Pátzcuaro con el fin de ser evaluados y eventualmente canalizados con las dependencias correspondientes para lograr apoyo y financiamiento. Los grupos con necesidad de más apoyo técnico tuvieron por medio año asesoría para dar continuidad a las tareas y conocer los avances registrados. 
CUADRO 1. Haz Crecer tú Comunidad

\begin{tabular}{|c|c|c|}
\hline Organización & Comunidad & Nombre del Proyecto \\
\hline Agua de San Gregorio & San Gregorio & $\begin{array}{l}\text { Embotelladora de agua "Agua y Vida de } \\
\text { San Gregorio" }\end{array}$ \\
\hline $\begin{array}{l}\text { "Jarhuata" Consultoría de } \\
\text { proyectos }\end{array}$ & Cuanajo & $\begin{array}{l}\text { Consultores para obtener fondos de los } \\
\text { organismos financieros }\end{array}$ \\
\hline Muebles Yarin & Cuanajo & $\begin{array}{l}\text { Nuevos Prototipos de Muebles de } \\
\text { Madera }\end{array}$ \\
\hline Juchari Uinapekua & $\begin{array}{l}\text { Santa Fe de la } \\
\text { Laguna }\end{array}$ & $\begin{array}{l}\text { Proyecto de Engorda de ganado } \\
\text { "Agrícola Ganadero" }\end{array}$ \\
\hline Kikiribu & Opopeo & $\begin{array}{l}\text { Grupo cooperativo de productores de } \\
\text { pollo de engorda y postura }\end{array}$ \\
\hline Bordados "El Rosario" & Opopeo & Bordados en punto de cruz \\
\hline Cultivarte & Pátzcuaro & $\begin{array}{l}\text { Elaboración de abonos orgánicos a base } \\
\text { de estiércol vacuno y lombriz roja } \\
\text { californiana. }\end{array}$ \\
\hline JOIMAC & Pátzcuaro & $\begin{array}{l}\text { Jóvenes impulsando el arte y la cultura } \\
\text { en Michoacán mediante la elaboración } \\
\text { de artesanías de materiales de reciclaje }\end{array}$ \\
\hline $\begin{array}{l}\text { Grupo Emprendedor Zirahuén } \\
\text { "GEZ" }\end{array}$ & Zirahuén & $\begin{array}{l}\text { Producción de Abono Orgánico y } \\
\text { Alimentos Orgánicos con el Método } \\
\text { Biointensivo }\end{array}$ \\
\hline Zirahongo & Zirahuén & Producción de Hongo Shitake \\
\hline Agua Chichinda & Zárate & Purificadora de Agua y Derivados \\
\hline RETEM & Morelia & Turismo Pedagógico \\
\hline RETEM & Morelia & Asesoría Académica \\
\hline RETEM & Morelia & Heladería Artesanal Italiana \\
\hline
\end{tabular}

Fuente: PROGRAMA HAZ CRECER TÚ COMUNIDAD, 2015.

Entre algunos de los resultados encontrados en el proyecto Haz Crecer Tú Comunidad se logró conformar al grupo Zirahongo, formado por estudiantes de un bachillerato técnico de educación media y que planteó generar una empresa para el cultivo de hongos seta. Por otra parte, se identificó a un grupo para promover una red estatal para la transformación educativa y crear una organización para impulsar el turismo pedagógico y la asesoría académica. En otra perspectiva, se conformó un grupo para llevar a cabo asesoría hacia la Juchari Uinapekua (engorda de toriles) y Kikiribu 
(engorda y postura de pollos). De igual modo, se conformó el grupo de bordados "El Rosario" y se creó un grupo de para la producción de abono orgánico a través de la lombriz roja californiana, y otro sobre para el reciclaje de la botella de agua.

$\mathrm{Si}$ bien estas experiencias resultaron prometedoras, es importantes señalar que no todas las ideas de proyectos fueron viables debido a que algunas propuestas insinuaron una mayor complejidad técnica y limitaciones para lograr el acceso al financiamiento. Por ejemplo, se constituyó un grupo en la comunidad de San Gregorio para utilizar agua de un manantial, el cual no logró obtener el permiso de la autoridades. Asimismo, un grupo denominado JOYMAC no logró conformarse como organización debido a la falta de participación entre sus miembros y otras experiencias no tuvieron el impacto esperado en sus comunidades debido a la desconfianza y el miedo existente en esos lugares.

\section{Gestión del desarrollo comunitario y municipal}

En otro contexto, el proyecto Gestión del Desarrollo Comunitario y Municipal se impulsó a través del municipio de Ecuandureo para obtener el apoyo financiero, organizativo y logístico del Ayuntamiento. La fase de sensibilización inició con una campaña de información entre la población del lugar a través de las autoridades comunitarias y se invitó a jóvenes que tuvieran la iniciativa para capacitarse y realizar trabajos de investigación y planeación con sus comunidades. Asimismo, con el Presidente Municipal se planteó la posibilidad de llevar a cabo el plan de desarrollo con base en los diagnósticos detectados y en las necesidades registradas en ese lugar (Beltrán; Brunner, 2014:48).

En una segunda fase, se conformó la idea de realizar una metodología basada en un diplomado para la creación de comunidades letradas a través de la colaboración de jóvenes gestores emprendedores y que pudieran capacitarse para elaborar proyectos en su comunidad. El diplomado se organizó con un total de seis módulos de trabajo y un módulo 
de presentación de resultados. Se trabajó en torno a los temas relacionados con la participación social, la regionalización y el desarrollo comunitario. Asimismo, se estableció que en todas las propuestas debía existir la visión del desarrollo sustentable y se proporcionaron elementos para la creación de una cartografía social comunitaria y sistematizar los resultados.

En este proyecto se trabajó con la consigna de aprender haciendo en el cual los participantes se integraron en equipos de trabajo por micro regiones y se clasificaron las propuestas conforme a temas básicos y prioritarios para el desarrollo comunitario y municipal. En la fase de desarrollo se registró la participación de cuatro equipos para trabajar en cuatro microrregiones del municipio conformadas por las localidades de Quiringúicharo, Las Fuentes y Ucácuaro, Moreno de Bravo y Ecuandureo. En el análisis se utilizó un enfoque de investigación mixto para recopilar información, analizar datos y generar una propuesta de interpretación con base en el uso de entrevistas, sondeos y observación en los lugares para facilitar el análisis social, económico y cultural en los poblados con mayor marginación.

Los resultados obtenidos en este proyecto revelaron una participación social importante al registrar la conclusión de los módulos de 23 de 27 jóvenes emprendedores y 12 proyectos de trabajo (véase CUADRO 2). Así, se logró desarrollar cuatro planes de desarrollo microrregional con proyectos específicos para cada población y se aportaron estrategias para el Plan de Desarrollo Municipal.

CUADRO 2. Gestión del Desarrollo Comunitario

\begin{tabular}{|c|c|c|}
\hline Micro-región & Localidades & Proyectos \\
\hline \multirow{3}{*}{1} & \multirow{3}{*}{$\begin{array}{l}\text { Quiringûicharo, La Soledad, } \\
\text { Rincón Grande y La Barranca, } \\
\text { Municipio de Ecuandureo. }\end{array}$} & Recicladora de plástico \\
\hline & & $\begin{array}{l}\text { Construcción de puentes sobre } \\
\text { caminos y canales agrícolas }\end{array}$ \\
\hline & & Procesadora de derivados de leche \\
\hline$\|$ & $\begin{array}{l}\text { Cabecera municipal y ejidos del } \\
\text { municipio de Ecuandureo }\end{array}$ & $\begin{array}{l}\text { Programa de Educación Ambiental } \\
\text { "Agricultor Responsable" }\end{array}$ \\
\hline
\end{tabular}




\begin{tabular}{|c|c|c|}
\hline & & Mercado Municipal de Ecuandureo \\
\hline \multirow{4}{*}{ III } & \multirow{4}{*}{$\begin{array}{l}\text { Ejido Moreno de Bravo, } \\
\text { Municipio de Ecuandureo }\end{array}$} & Baños secos \\
\hline & & $\begin{array}{c}\text { Camas de cultivo } \\
\text { biointensivo }\end{array}$ \\
\hline & & $\begin{array}{c}\text { Perforación de pozo profundo } \\
\text { para agua potable }\end{array}$ \\
\hline & & $\begin{array}{c}\text { Taller de transformación de } \\
\text { leche de cabra y vaca }\end{array}$ \\
\hline \multirow{3}{*}{ IV } & \multirow{3}{*}{$\begin{array}{l}\text { Las Fuentes y Ucácuaro, } \\
\text { Municipio de Ecuandureo }\end{array}$} & $\begin{array}{c}\text { Transformación de derivados } \\
\text { de leche de cabra }\end{array}$ \\
\hline & & $\begin{array}{c}\text { Mejoramiento y rehabilitación de } \\
\text { clínica rural en la localidad de } \\
\text { Ucácuaro en el Municipio de } \\
\text { Ecuandureo }\end{array}$ \\
\hline & & Siembra experimental de Leucaena \\
\hline
\end{tabular}

Fuente: PROGRAMA HAZ CRECER TÚ COMUNIDAD, 2015.

Entre las iniciativas impulsadas destacaron los proyectos sobre educación ambiental para el municipio, la construcción del mercado municipal en Ecuandureo y la elaboración de baños secos ecológicos, así como la creación de camas de cultivo biointensivo para hortalizas en cada unidad doméstica del Ejido de Moreno de Bravo. Asimismo, se logró conformar un proyecto para la construcción de un puentes sobre caminos y canales agrícolas y la construcción de una planta recicladora de plásticos en el ejido de Quiringuicharo. Por otra parte, se trabajó en la conformación de un proyecto de siembra experimental de leucaena (un arbusto de forraje para animales) en Ucácuaro y un plan para la transformación de derivados de leche de cabra para la producción de quesos, chongos, cajetas y dulces en Rincón Grande y en los ejidos de Moreno de Bravo y Las Fuentes (CREFAL, 2012).

Todos los proyectos se presentaron a la comunidad a través de los representantes municipales y se convocó a instituciones privadas, grupos y organizaciones de la sociedad civil para visibilizar la importancia de crear proyectos para el desarrollo comunitaria desde una perspectiva solidaria. Si bien, se pude afirmar que existió un avance importante en la promoción del 
tema en esta región, es necesario reconocer que los impactos de estas acciones son limitadas debido a la escases de recursos y la falta de coordinación interinstitucional observada entre autoridades para llevar a cabo estas propuesta de una forma más integral.

\section{Producción orgánica sustentable y alimentación sana}

Considerando que la degradación del suelo por el uso de fertilizantes químicos y los efectos del cambio climático global causan problemas en la mayoría de las comunidades rurales, así como debido a que la calidad de los alimentos no es la adecuada y genera problemas de salud y obesidad en las personas, el CEDECOSU creó el proyecto Producción Orgánica Sustentable y Alimentación Sana, conocido como POSIBLE a través de la conformación de un diplomado como estrategia para comunicar entre la comunidad de la zona lacustre en Pátzcuaro.

El Diplomado se impulsó hacia familias de escasos recursos para conocer e implementar formas orgánicas y sustentables de producción agrícola, sobre todo de horticultura mediante el cultivo biointensivo en espacios reducidos, la producción de abonos orgánicos para los huertos y las milpas y la elaboración de alimentos ricos en elementos nutritivos para el autoconsumo y la comercialización solidaria. El programa obtuvo el apoyo financiero de la Embajada de Alemania en México y se coordinó con los temas del Centro de Educación y Capacitación para el Desarrollo Sustentable (CREDES) del gobierno federal.

La organización del diplomado se estructuró en cuatro módulos de trabajo sobre la elaboración de lombricultura, huertos biointensivos, alimentación sana y emprendimiento para negocios ecológicos. En la fase de sensibilización, el proyecto inició como acciones aisladas y una metodología exploratoria para difundir la propuesta entre la comunidad e impulsar la participación comunitaria en la región.

En la fase de desarrollo, se logró trabajar con 12 grupos familiares en 11 localidades rurales de los municipios de Salvador Escalante y Pátzcuaro. 
Hacia diciembre de 2012, los rendimientos registrados fueron importantes debido a que se encontró un interés para desarrollar las bases de los proyectos y se logró crear confianza y responsabilidad familiar. Los resultados del proyecto mostraron la construcción de 12 camas y hacia el 2013, se alcanzó un total de 22 camas instaladas. Se registró una producción de 846.5 kg de humus y de 362 litros de lixiviado; asimismo, se impartieron 112 asesorías familiares y 7 talleres de capacitación (BELTRÁN, BRUNNER, 2014).

La mitad de las familias que participaron en el módulo de Lombricultura, siguieron asistiendo al módulo de huertos biointensivos que impartió el CREDES y donde aumentó la asistencia de famílias (véase CUADRO 3). Cabe mencionar que los huertos biointensivos se fertilizan con el abono orgánico producido por las lombrices y ofrecen la posibilidad de cultivar una abundancia de hortalizas en pequeños espacios con el beneficio adicional de enriquecer los suelos poco fértiles. Además, con los huertos biointensivos se logra simultáneamente la conservación del medio ambiente, el mejoramiento en la calidad de la alimentación de las comunidades y la recuperación de tradiciones y saberes ancestrales.

CUADRO 3. Producción Orgánica Sustentable y Alimentación Sana (POSIBLE)

\begin{tabular}{|c|c|c|}
\hline \multicolumn{2}{|c|}{ Proyecto } & \multicolumn{2}{c|}{ Lugar } \\
\hline $\begin{array}{c}\text { Producción de Abono } \\
\text { Orgánico a través de la } \\
\text { Lombriz Roja Californiana }\end{array}$ & Salvador Escalante. & $\begin{array}{c}18 \text { camas de lombricultura } \\
\text { beneficiando a } 12 \text { familias }\end{array}$ \\
\hline Huertos Biointensivos & Salvador Escalante & $\begin{array}{c}25 \text { huertos biointensivos y el } \\
\text { número de camas de } \\
\text { lombricultura } 28 .\end{array}$ \\
\hline
\end{tabular}

Fuente: PROGRAMA HAZ CRECER TÚ COMUNIDAD, 2015.

En el municipio de Salvador Escalante se realizaron 16 proyectos en las comunidades Santa Clara del Cobre, Zirahuén y Opopeo de las que se obtuvieron 25 huertos biointensivos y para el 2014, se anotó un total de 28 camas de lombricultura. Asimismo, se logró obtener cultivos como lechuga, jitomate, acelgas, rábanos, betabel, zanahoria, cilantro, chile serrano, 
calabaza, repollo, cebolla, frijol, garbanzo, árnica, entre otros productos orgánicos.

Hacia el final de 2014, se registraron 6 escuelas que mostraron interés para organizar cursos de verano y crear un recetario de comida para el ciclo escolar. Por otra parte, también se mostraron retos importantes para mejorar el modelo del diplomado y se detectó la falta de motivación en las familias debido a los cambios que se presentan en el financiamiento por parte de las organizaciones e instituciones participantes. El proyecto POSIBLE se puede considerar como experiencia exitosa en la medida de que se logró compartir conocimiento, capacidades para la integración familiar y, sobre todo, lograr métodos y técnicas de cultivo para formar emprendedores desde una perspectiva social, consciente y para el beneficio de la cohesión comunitaria de la región Pátzcuaro.

\section{Reflexiones finales}

Los problemas de desempleo, pobreza, inseguridad y exclusión social que se presentan en México en las última dos décadas como consecuencia de la profundización y efectos de las políticas neoliberales entre la población en los últimos años, particularmente en el Estado de Michoacán, han contribuido al aumento de los problemas sociales y la pérdida de confianza entre los jóvenes y adultos hacia las políticas y las acciones de gobierno. El emprendimiento social constituye una opción para resolver problemas públicos específicos de la comunidad y una esperanza para poner en marcha un modelo de trabajo más humano y sustentable. Asimismo, constituye un eje para la rearticulación entre Estado y sociedad desde una perspectiva incluyente, democrática y con valor público.

El trabajo que realizó el CENTRO DE DESARROLLO COMUNITARIO SUSTENTABLE (CEDECOSU) del CREFAL logró, de alguna manera, la coordinación de esfuerzos educativos para generar alternativas hacia el desarrollo comunitario. Los proyectos Haz crecer tú comunidad, Gestión del Desarrollo Comunitario y Producción Orgánica Sustentable desarrollados 
entre 2012 y 2014, revelaron la necesidad de impulsar procesos de aprendizaje para la creación de empresas sociales y solidarias, principalmente en la región Pátzcuaro-Zirahuén y constituyen ejemplos representativos para motivar a la comunidad y enfrentar los problemas de exclusión social y educativa.

En este proceso, es importante reflexionar acerca de cuáles son los mecanismos que permiten impulsar el desarrollo educativo de las personas en los proyectos productivos; así como aclarar qué tipo de asesorías, materiales de apoyo educativo se requieren para mejorar las estrategias y la misión del programa general; y finalmente, cómo motivar la colaboración interinstitucional de los proyectos para lograr un mayor financiamiento y credibilidad en las tareas que advierte el emprendedurismo en esta región.

En primer lugar, y de acuerdo con los objetivos de la Agenda 2030, de la ORGANIZACIÓN DE LAS NACIONES UNIDAS (ONU, 2015) que tienen como propósito transformar nuestro mundo, es necesario revalorar las políticas hacia el desarrollo para incentivar los sistemas productivos locales y apoyar las iniciativas de cambio que se registran las comunidades rurales. Con esto será posible no sólo atender los rezagos históricos en la educación, el empleo y la salud, sino establecer otras formas de organización comunitaria basadas en el desempeño de sus valores, normas y procesos de movilización desde y para la comunidad.

En segundo lugar, lograr el desarrollo de proyectos de emprendimiento exitosos y con valor público, requiere la formación de una red de esfuerzos humanos y materiales para motivar procesos capacitación y consulta, así como generar la infraestructura tecnológica requerida para incidir en la construcción de capacidades políticas y administrativas de los municipios. Estas acciones permitirán generar capital social en su propio beneficio, así como evitar el clientelismo y asistencialismo como únicas estrategias de participación para el desarrollo social. En algunas experiencias, se detectó que es necesario contar con mayor voluntad de las autoridades para comprometerse con la gestión del desarrollo comunitario y evitar prácticas 
clientelistas que dañen la transparencia, aumenten el burocratismo y la corrupción en el manejo de los recursos.

En tercer lugar, es importante fortalecer los programas de formación educativa con base en las necesidades que tienen las propias comunidades y desde su ámbito cultural. En los proyectos referidos se detectó que algunos grupos se enamoraron de sus propuestas y se inició la revisión metodológica de los programas para avanzar hacia otra dirección utilizando la experiencia de los promotores, empresarios sociales y participantes en los diplomados.

Se puede afirmar que la utilización de las ideas de la comunidad es, además, un factor excelente en el arraigo del proyecto. En este sentido, la apropiación de los proyectos por parte de la comunidad se fortalece con la organización de ferias y otros eventos cívicos de gran escala para la presentación de los resultados que tienen el efecto de producir orgullo en los participantes y el deseo de seguir trabajando. Asimismo, la transformación de los proyectos en cursos y diplomados es un factor de éxito porque la certificación oficial de conocimientos y habilidades adquiridas durante el trabajo legitima y crea valor público y construye ciudadanía entre la comunidad.

Por último, no está de más señalar que los retos que se presentan para lograr una colaboración efectiva entre organizaciones e instituciones, revelan la necesidad movilizar mayores recursos económicos para potenciar el desarrollo local en esta región, combatir los problemas sociales (migración, desempleo, pobreza, entre otros) y crear las condiciones de vida que requieren las comunidades en esta entidad afectada por la crisis económica en México. 


\section{Referencias}

ANDER-EGG, E. Cómo elaborar un proyecto. Guía para diseñar proyectos sociales y culturales, Buenos Aires, Lumen-Hvmanitas, 2000.

BAÑOS, B. Participación social y desarrollo de la comunidad en Loma Larga, Pinotepa Nacional, Oaxaca, México, Tesis de Maestría en Ciencias, Colegio de Posgraduados del Estado de México, 2012.

BELTRÁN, V. Sistematización de experiencias de aprendizaje en proyectos de desarrollo comunitario sustentable del CREFAL: Ia documentación como producto final, Ponencia. 5to Congreso Internacional de Desarrollo Comunitario, Pátzcuaro, Michoacán, 2015.

BELTRÁN, V.; BRUNNER, I. El Centro de Desarrollo Comunitario Sustentable del CREFAL, Revista Decisio No. 39, Centro de Cooperación Regional para la Educación de Adultos en América Latina y el Caribe (CREFAL), septiembrediciembre, 2014.

BOUZADA, X. Elementos teóricos relativos al desarrollo comunitario local y a su práctica en la comunidad autónoma de Galicia, en Revista de Sociología, v. 45, 1994, p. 81-100, Disponible en: http://papers.uab.cat/article/view/v45bouzada. Recuperado el 23 de mayo de 2020.

CASTELAR, M. Las políticas públicas y sus visión de la economía social y solidaria en Argentina, en Revista Mexicana de Ciencias Políticas y Sociales No. 227, FCPyS-UNAM, mayo-agosto de 2016, pp. 349-378.

CENTRO DE COOPERACIÓN REGIONAL PARA LA EDUCACIÓN DE ADULTOS EN AMÉRICA LATINA Y EL CARIBE (CREFAL), Documento Base del Programa Michoacán Letrado (DBML), Michoacán, México, 2011.

CENTRO DE COOPERACIÓN REGIONAL PARA LA EDUCACIÓN DE ADULTOS EN AMÉRICA LATINA Y EL CARIBE (CREFAL). Informes de gestión del Proyecto Diplomado Haz crecer tu comunidad, México, Centro de Cooperación Regional para la Educación de Adultos en América Latina y el Caribe (CREFAL), junio, 2015.

CHALCATL, R. Emprendimiento social: apuntes para su estudio en México, México, Tesis de Licenciatura en Administración-UNAM, 2015.

CHONG-GONZÁLEZ, G. El emprendimiento como agente potenciador del desarrollo económico local en el espacio rural, Desarrollo Económico, Bolivia, Vol. 4, No. 11, 2017.

CORAGGIO, J. El papel de la economía social y solidaria en la estrategia de inclusión social, Revista Decisio, mayo-agosto, México, CREFAL, 2011. 
Recuperado 4 de enero de 2020

http://www.crefal.edu.mx/decisio/images/pdf/decisio_29/decisio29_saber4.p df

DIARIO OFICIAL DE LA FEDERACIÓN. Ley y Programa de Fomento a la Economía Social, 2015.

http://www.diputados.gob.mx/LeyesBiblio/pdf/LESS_120419.pdf

GOBIERNO DEL ESTADO DE MICHOACÁN, Plan de Desarrollo Integral del Estado de Michoacán 2015-2021. Informes regionales, 2015. Recuperado el 22 de mayo, 2020, http://foros.michoacan.gob.mx/region-7/

INCHÁUSTEGUI, T. Desarrollo local, en Bokser, Judith, Laura Baca, E†. Al., Léxico de la Política, México, Fondo de Cultura Económica, 2001.

INSTITUTO NACIONAL DE GEOGRAFÍA Y ESTADÍSTICA (INEGI), Información básica de Michoacán de Ocampo, consulta 20 de mayo, 2020.

KLISKBERG, B. ¿Cómo impulsar la participación comunitaria? Clave estratégica para enfrentar la exclusión y la desigualdad, y devolver la dignidad, Argentina, Red Latinoamericana de Universidades por el Emprendedurismo Social, 2015.

LEÓN, B. Entre la redención y la conducción: el combate a la pobreza en México 1970-2012, México, Editorial Fontamara, 2014.

MONZÓN, J.L; DEFOURNY, (Ed). (1992), La Economía Social: entre Economía Capitalista y Economía Pública, CIRIEC-España, Editorial Valencia, 1992.

MORENO-BRID, J.; ROS, J. Desarrollo y crecimiento en la economía mexicana. Una perspectiva histórica, México, Fondo de Cultura Económica, 2010.

ORGANIZACIÓN DE LAS NACIONES UNIDAS (ONU). Resolución aprobada por la Asamblea General el 25 de septiembre de 2015. Transformar nuestro mundo: la Agenda 2030 para el Desarrollo Sostenible, ONU, octubre 2015, Disponible en: http://www.un.org/es/comun/docs/?symbol=A/RES/70/1

PALACIOS, G. Emprendimiento social: integrando a los excluidos en el ámbito rural, en Revista de Ciencias Sociales, Venezuela, Vol. XVI, No. 4, octubrediciembre, 2010, pp. 579-590.

Recebido em 17 de março de 2020.

Aprovado em 27 de julho de 2020. Publicado em 19 de agosto de 2020. 